\title{
NOTE ON SOME PARTITION IDENTITIES
}

\section{CARLITZ}

1. Introduction. In a recent paper, Newman [4] states the formulas

$$
\begin{aligned}
& \sum_{0}^{\infty} p_{2}(11 m+10) x^{n}=\sum_{1}^{\infty}\left(1-x^{11 n}\right)^{2} \\
& \sum_{0}^{\infty} p_{1}(11 m+20) x^{n}=-11 \prod_{1}^{\infty}\left(1-x^{11 n}\right)^{4}, \\
& \sum_{0}^{\infty} p_{2}(17 m+24) x^{n}=-\prod_{1}^{\infty}\left(1-x^{17 n}\right)^{2} \\
& \sum_{0}^{\infty} p_{6}(31 m+240) x^{n}=961 \prod_{1}^{\infty}\left(1-x^{31 n}\right)^{6}
\end{aligned}
$$

where

$$
\prod_{n=1}^{\infty}\left(1-x^{n}\right)^{k}=\sum_{m=0}^{\infty} p_{k}(m) x^{m} .
$$

We wish to point out that results of this kind can be obtained in a very elementary way, namely, by using a method employed by Ramanujan in proving the formula $p(5 m+4)=0(\bmod 5)$ (see for example [2, p. 87]). We shall prove the following formulas. Let $r$ be prime. If $r \equiv 3(\bmod 4), r>3$, then

$$
\sum_{m=0}^{\infty} p_{2}\left(r m+r_{0}\right) x^{m}=\prod_{n=1}^{\infty}\left(1-x^{r n}\right)^{2},
$$

where $r_{0}=\left(r^{2}-1\right) / 12$.

If $r \equiv 3(\bmod 4), r \geqq 3$, then

$$
\sum_{m=0}^{\infty} p_{6}\left(r m+r_{1}\right) x^{m}=r^{2} \prod_{n=1}^{\infty}\left(1-x^{r n}\right)^{6},
$$

where $r_{1}=\left(r^{2}-1\right) / 4$.

If $r \equiv 5(\bmod 6)$, then

$$
\sum_{m=0}^{\infty} p_{4}\left(r m+r_{2}\right) x^{m}=-r \prod_{n=1}^{\infty}\left(1-x^{r n}\right)^{4},
$$

where $r_{2}=\left(r^{2}-1\right) / 6$.

Received by the editors October 27, 1952. 
If $r \equiv 5(\bmod 12)$, then

$$
\sum_{m=0}^{\infty} p_{2}\left(r m+r_{0}\right) x^{m}=-\prod_{n=1}^{\infty}\left(1-x^{r n}\right)^{2},
$$

where $r_{0}=\left(r^{2}-1\right) / 12$.

It is clear that (1.1) is contained in (1.5), (1.2) in (1.7), (1.3) in (1.8), (1.4) in (1.6); the case $r=5$ of (1.7) occurs in [3]. We also remark that $(1.5), \cdots,(1.8)$ can be put in somewhat sharper form; for example in place of (1.5) we can state

$$
\sum_{m=0}^{\infty} p_{2}\left(r^{2} m+r_{0}\right) x^{m}=\prod_{n=1}^{\infty}\left(1-x^{n}\right)^{2}=\sum_{m=0}^{\infty} p_{2}(m) x^{m}
$$

In other words

$$
p_{2}\left(r^{2} m+r_{0}\right)=p_{2}(m) ; \quad p_{2}\left(r m+r_{0}\right)=0 \text { for } \quad r k m .
$$

Similar results hold for the other functions.

2. Proof of (1.5). By Euler's formula

$$
x^{s} \prod_{n=1}^{\infty}\left(1-x^{n}\right)^{2}=\sum_{h, k=-\infty}^{\infty}(-1)^{h+k} x^{s+h(3 h+1) / 2+k(3 k+1) / 2},
$$

where $s$ is to be assigned. The exponent on the right is divisible by $r$ provided

$$
(6 h+1)^{2}+(6 k+1)^{2}+2(12 s-1) \equiv 0(\bmod r) .
$$

If we take $s$ as the least positive integer such that $12 s \equiv 1(\bmod r)$, then by the hypothesis on $r$ it is clear that (2.2) implies $r \mid 6 h+1$, $r \mid 6 k+1$. Thus with a little manipulation (2.1) yields

$$
\sum_{m=0}^{\infty} p_{2}(r m+r-s) x^{m}=x^{e} \prod_{n=1}^{\infty}\left(1-x^{r n}\right)^{2}
$$

where

$$
e=\frac{12 s-1}{12 r}+\frac{r}{12}-1
$$

Since

$$
r e+r-s=\frac{12 s-1}{12}+\frac{r^{2}}{12}-s=\frac{r^{2}-1}{12},
$$

(1.5) follows at once. 
3. Proof of (1.6). Using Jacobi's formula we have

$$
\begin{aligned}
x^{3} \prod_{n=1}^{\infty}(1- & \left.x^{n}\right)^{6} \\
& =\sum_{h, k=0}^{\infty}(-1)^{h+k}(2 h+1)(2 k+1) x^{s+h(h+1) / 2+k(k+1) / 2} .
\end{aligned}
$$

The exponent on the right is divisible by $r$ provided

$$
(2 h+1)^{2}+(2 k+1)^{2}+2(4 s-1) \equiv 0(\bmod r) .
$$

If we choose $s$ as the least positive integer such that $4 s \equiv 1(\bmod r)$, (3.2) implies $r|2 h+1, r| 2 k+1$. Thus, very much as above, (3.1) yields

$$
\sum_{m=0}^{\infty} p_{6}(r m+r-s) x^{m}=r^{2} x^{e} \prod_{n=1}^{\infty}\left(1-x^{r n}\right)^{6},
$$

where

$$
e=\frac{8 s-1}{8 r}+\frac{r}{4}-1
$$

Since

$$
r e+r-s=\frac{8 s-1}{8}+\frac{r^{2}}{4}-s=\frac{r^{2}-1}{4}
$$

(1.6) follows at once.

4. Proof of (1.7). Using Euler's and Jacobi's formula we have

(4.1) $x^{8} \prod_{n=1}^{\infty}\left(1-x^{n}\right)^{4}=\frac{1}{2} \sum_{h, k=-\infty}^{\infty}(-1)^{n+k}(2 k+1) x^{0+h(8 h+1) / 2+k(k+1) / 2}$.

The exponent on the right is divisible by $r$ provided

$$
(6 h+1)^{2}+3(2 k+1)^{2}+4(6 s-1) \equiv 0(\bmod r) .
$$

We choose $s$ as the least positive integer such that $6 s \equiv 1(\bmod r)$. Since -3 is a quadratic nonresidue of $r$, it follows from (4.2) that $r|6 h+1, r| 2 k+1$. A little attention must now be paid to the sign in the right member of (4.1). We find without much trouble that (4.1) implies

$$
\sum_{m=0}^{\infty} p_{4}(r m+r-s) x^{m}=-r x^{e} \prod_{n=1}^{\infty}\left(1-x^{r n}\right)^{4},
$$

where 


$$
e=\frac{s-1}{6 r}+\frac{r}{6}-1
$$

Since

$$
r e+r-s=\frac{s-1}{6}+\frac{r^{2}}{6}-s=\frac{r^{2}-1}{6},
$$

it is evident that (4.3) reduces to (1.7).

5. Proof of (1.8). We return to (2.1) and (2.2). Since $r \equiv 1(\bmod 4)$ we can no longer assert that $r|6 h+1, r| 6 k+1$, but only that $(6 h+1)^{2}$ $+(6 k+1)^{2} \equiv 0(\bmod p)$. Changing the notation slightly, consider

$$
h=a u-b v, \quad k=a v+b u,
$$

where $r=a^{2}+b^{2}$ and $h \equiv k \equiv 1(\bmod 6)$. Since $r \equiv 5(\bmod 12)$, we may suppose that $a \equiv 1, b \equiv \pm 2(\bmod 6)$. If $b \equiv 2(\bmod 6)$, consider

$$
r h^{\prime}=-\left(a^{2}-b^{2}\right) h-2 a b k, \quad r k^{\prime}=-2 a b h+\left(a^{2}-b^{2}\right) k .
$$

Then by (5.1), (5.2) reduces to $h^{\prime}=-a u-b v, k^{\prime}=-b u+a v$, so that $h^{\prime}$ and $k^{\prime}$ are integers; moreover $h^{\prime 2}+k^{\prime 2}=h^{2}+k^{2}$. In the next place (5.2) implies

$$
\begin{array}{ll}
5 h^{\prime} \equiv 3 h-4 k \equiv-1, & h^{\prime} \equiv 1(\bmod 6), \\
5 k^{\prime} \equiv-4 h+3 k=-1, & k^{\prime} \equiv 1 .
\end{array}
$$

On the other hand (5.2) implies

$$
h^{\prime} \equiv-h, \quad k^{\prime} \equiv k(\bmod 4) .
$$

It follows that the terms in the right member of (2.1) corresponding to $(h, k)$ and $\left(h^{\prime}, k^{\prime}\right)$ cancel.

Next, if $b \equiv-2(\bmod 6)$, we change all signs in the right members of (5.2). The details are much as before; in particular (5.3) becomes $h^{\prime} \equiv h, k^{\prime} \equiv-k(\bmod 4)$. Thus once again corresponding terms cancel.

Now consider a pair $(h, k)$ with $h^{2}+k^{2}=m$, where $m$ is fixed, $r \mid m$, $h \equiv k \equiv 1(\bmod 6)$. Suppose first $r \nmid h$. Then if $r \nmid h^{\prime}$, it is clear from the above that the corresponding terms in (2.1) cancel. On the other hand, when $r \mid h$, then it follows from the above discussion that we can simultaneously consider the correspondence (5.2) together with the second correspondence $(b \equiv-2)$. In other words we have in this case $(r \mid h)$ a $(2,1)$ correspondence. Returning to $(2.1)$ we see that

$$
\sum_{m=0}^{\infty} p_{2}(r m+r-s)=-x^{e} \prod_{n=1}^{\infty}\left(1-x^{r n}\right)^{2},
$$


where $e$ is determined by (2.3). The proof of (1.8) is now completed in exactly the same way as in (1.5).

6. Another formula. Newman also states the formula

$$
\sum_{m=0}^{\infty} p_{s}(5 m) x^{m}=\prod_{n=1}^{\infty}\left(1-x^{n}\right)^{6}\left(1-x^{5 n}\right)^{-1},
$$

which he notes had been found (but not published) by D. H. Lehmer. It may be of interest to point out that (6.1) can be obtained easily from the identity.

$$
\prod_{n=1}^{\infty} \frac{\left(1-x^{n}\right)^{5}}{1-x^{5 n}}=1-5 \sum_{m=1}^{\infty}\left(\frac{m}{5}\right) \frac{x^{m}}{1-x^{m}} .
$$

The formula (6.2) is due to Ramanujan; Bailey [1] showed recently that it is a consequence of well knpwn formulas for the Weierstrass elliptic functions.

Since the right member of (6.2) equals

$$
1-5 \sum_{m, r=1}^{\infty}\left(\frac{m}{5}\right) m x^{m r}
$$

it follows that

$$
\begin{aligned}
\sum_{m=0}^{\infty} p_{5}(5 m) x^{5 m} \prod_{n=1}^{\infty}\left(1-x^{5 n}\right)^{-1} & =1-5 \sum_{m=1}^{\infty}\left(\frac{m}{5}\right) \frac{x^{5 m}}{1-x^{5 m}} \\
& =\prod_{n=1}^{\infty}\left(1-x^{5 n}\right)^{5}\left(1-x^{25 n}\right)^{-1}
\end{aligned}
$$

Replacing $x^{5}$ by $x$ we get (6.1).

\section{REFERENCES}

1. W. N. Bailey, A further note on two of Ramanujan's formulae, Quart. J. Math. Oxford Ser. (2) vol. 3, pp. 158-160.

2. G. H. Hardy, Ramanujan, Cambridge, 1940.

3. D. Kruyswijk, On some well-known properties of the partition function and Euler's infinite product, Nieuw Archief voor Wiskunde vol. 23 (1950) pp. 97-107.

4. M. Newman, Remarks on some modular identities, Trans. Amer. Math. Soc. vol. 73 (1952) pp. 313-320.

DUKE UNIVERSITY 